\title{
Inhaled tobramycin solution-associated recurrent eosinophilia and severe persistent bronchospasm in a patient with cystic fibrosis: a case report
} Roberto P Santos, Emad Awa and Ran D Anbar*

Address: Department of Pediatrics, University Hospital, State University of New York Upstate Medical University, 750 East Adams Street, Syracuse, New York 13210, USA

Email: Roberto P Santos - Roberto.Santos@UTSouthwestern.edu; Emad Awa - emadawa@hotmail.com; Ran D Anbar* - anbarr@upstate.edu

* Corresponding author

Published: 2 March 2007

BMC Pediatrics 2007, 7:1I doi:10.1 |86/147|-2431-7-II
Received: 23 August 2006

Accepted: 2 March 2007

This article is available from: http://www.biomedcentral.com/I47I-243I/7/II

(C) 2007 Santos et al; licensee BioMed Central Ltd.

This is an Open Access article distributed under the terms of the Creative Commons Attribution License (http://creativecommons.org/licenses/by/2.0), which permits unrestricted use, distribution, and reproduction in any medium, provided the original work is properly cited.

\begin{abstract}
Background: Delivery of tobramycin by inhalation to the lungs of patients with cystic fibrosis (CF) who are infected with Pseudomonas aeruginosa has been proven to be effective and safe. The aerosol administration allows high concentrations of tobramycin to be delivered to the site of infection with limited systemic absorption. In rare patients, systemic absorption of inhaled tobramycin may be significant enough to produce toxic effects, such as renal and vestibular toxicities.
\end{abstract}

Case presentation: We report a patient with CF who developed recurrent eosinophilia and severe persistent bronchospasm following repeated administration of preservative-free tobramycin by inhalation, beginning at 16 months of age. Also, he developed similar signs and symptoms when he was administered tobramycin intravenously on one occasion at $5 \mathrm{I} / 2$ years. The patient had a history of environmental allergies. Temporal sequence of his signs and symptoms after each administration of tobramycin (similar to re-challenge testing), and his improvement after discontinuation of the drug strongly suggest an adverse drug reaction.

Conclusion: Hypersensitivity reaction should be considered in patients who develop recurrent eosinophilia and deterioration of pulmonary function following the use of tobramycin by inhalation or by intravenous administration.

\section{Background}

The delivery of tobramycin by inhalation to the lungs of patients with cystic fibrosis (CF) who are infected with Pseudomonas aeruginosa has been proven to be effective and safe [1]. The aerosol administration allows high concentrations of tobramycin to be delivered to the site of infection with limited systemic absorption. The portion of inhaled tobramycin available for systemic absorption includes approximately $10 \%$ of the total dose that is deliv- ered to the lungs, and some that gets to the oropharynx and eventually swallowed [1].

There is poor systemic absorption of aerosolized tobramycin into the lungs among patients with $\mathrm{CF}$, and its absorption from alveolar space into the pulmonary circulation is rate-limited [2]. Several studies have consistently documented low serum concentrations of tobramycin following its aerosolized administration [1]. Rarely, renal [3] and vestibular toxicities [4] have been described following 
inhaled tobramycin. A single case report describes development of a rash following inhaled tobramycin, in a patient who had developed a similar rash following a course of intravenous gentamicin and piperacillin-tazobactam [5]. These examples illustrate that systemic absorption of inhaled tobramycin may be significant enough to produce toxic effects among patients with CF. Inhaled tobramycin has been associated with cough and alteration of voice $[6,7]$. Development of acute bronchospasm has been associated with use of intravenous preparations of tobramycin for aerosol administration. This effect is thought to be related to antioxidants and preservatives contained within these preparations [1]. Thus, bronchospasm is less likely to occur with use of TOBI ${ }^{\circledast}$ $[6,7]$, the preservative free tobramycin solution for inhalation (TSI) that has been licensed by the United States Food and Drug Administration.

Eosinophilia has been reported to be associated with intravenous administration of tobramycin, including a patient treated for Gram-negative bacteremia [8], and among nine of 88 patients who received tobramycin in combination with cefamandole for peritonitis [9]. Furthermore, a patient with end-stage renal disease who was treated for sepsis with parenteral tobramycin and vancomycin developed persistent fever, eosinophilia and rash. Although temporal sequence had suggested vancomycin as the probable cause [10], the possible contribution of tobramycin could not be totally excluded.

We report a patient with CF who developed recurrent eosinophilia and severe persistent bronchospasm following repeated administration of $\mathrm{TOBI}^{\oplus}$.

\section{Case Presentation}

The patient was diagnosed as having $\mathrm{CF}$, based on prenatal testing that was performed because he had a brother with CF. His CF transmembrane conductance regulator (CFTR) protein genetic analysis showed homozygosity for the $\Delta \mathrm{F} 508$ mutation. At a year of age, his treatments included pancreatic enzymes for his pancreatic insufficiency; a multivitamin; nebulized albuterol and cromolyn sodium in the treatment of occasional coughing and wheezing that were thought to be attributable to hyperre- active airways; and daily dornase alfa, which has been used routinely as preventive therapy in infants at our Cystic Fibrosis Center since 1996.

At 16 months of age, the patient presented with wheezing, and was found to harbor Pseudomonas aeruginosa in his deep throat culture for the first time. He was prescribed a one-month course of preservative-free TSI, $150 \mathrm{mg} / 2.5$ $\mathrm{mL}$ twice daily by nebulization. With this therapy, his cough and wheezing improved, only to worsen when the TSI was discontinued. Therefore, he was started on every other month administration of TSI that appeared to be recurrently associated with improvement in his respiratory status, even though Pseudomonas was not again documented in his throat cultures at that time. A year later his TSI dose was increased to $300 \mathrm{mg} / 5 \mathrm{~mL}$ twice daily.

At 2 years of age, as part of a routine screen, the patient was noted to have an elevated serum IgE level of $843 \mathrm{IU} /$ $\mathrm{mL}$ (normal range, 2-35 $\mathrm{IU} / \mathrm{mL}$ ), and his eosinophil count $(11 \%)$ was increased several fold (Table 1). The possibility of allergic bronchopulmonary aspergillosis was considered. However, anti-Aspergillus antibodies (< 1:8 by complement fixation) as well as skin scratch testing for Aspergillus were negative. As the patient's eosinophilia persisted (15-20\%), further investigations over the subsequent three years included a re-check of Aspergillus antibody titers (including A. fumigatus, A. flavus, and A. niger), stool sample for ova and parasites (including wet mount, permanent stain and modified acid fast stain), Giardia antigen in stool, and Toxocara canis antibody levels; all were negative. His radioallergosorbent test (RAST) for common allergens in the northeastern part of the United States showed low level response to Alternaria alternata (77\%) and to D. farinae (71\%). A chest radiograph was clear.

The patient continued to suffer from recurrent wheezing and therefore was prescribed nebulized budesonide, oral montelukast, and infrequent 3-5 day bursts of oral prednisolone. When the patient was nearly 5 years old he began performing pulmonary function testing (PFT) on a routine basis, and demonstrated normal lung function, without improvement following bronchodilator adminis-

Table I: Summary of TSI administration, pulmonary status, and hematologic profiles before 5 years

\begin{tabular}{|c|c|c|c|c|c|}
\hline & Before TSI Therapy & $\begin{array}{c}\text { TSI started } 150 \mathrm{mg} / 2.5 \\
\mathrm{~mL} \text { twice daily }\end{array}$ & $\begin{array}{c}\text { On TSI } 150 \mathrm{mg} / 2.5 \mathrm{~mL} \\
\text { twice daily }\end{array}$ & $\begin{array}{l}\text { TSI Increased to } 300 \mathrm{mg} / \\
5 \mathrm{~mL} \text { twice daily }\end{array}$ & 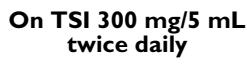 \\
\hline Age & 6 months & 16 months & 2 years & $2 \mathrm{I} / 2$ years & 4 years \\
\hline Pulmonary Status & No wheezing & $\begin{array}{l}(+) \text { wheezing; | } \text { It }^{(+)} \text {throat } \\
\text { culture for } P \text {. aeruginosa }\end{array}$ & $(+)$ wheezing & $(+)$ wheezing & $(+)$ wheezing \\
\hline WBC $\left(/ \mathrm{mm}^{3}\right)$ & 15.2 & NA & 9.1 & 12 & 11.2 \\
\hline Eosinophils (\%) & 1 & NA & 11 & 15 & 20 \\
\hline $\operatorname{IgE}(\mathrm{IU} / \mathrm{mL})$ & NA & NA & 843 & NA & 299 \\
\hline
\end{tabular}

Abbreviations: TSI, tobramycin solution for inhalation; WBC, white blood cell count; NA, not available 
tration, during a month while he was off TSI (Table 2). As he had not been found to harbor Pseudomonas for more than three years, it was decided to stop his every other month TSI.

At $51 / 2$ years the patient developed purulent nasal drainage, coughing and wheezing. At that time, his deep throat culture revealed Staphylococcus aureus and Serratia marcescens. As he did not respond to therapy with oral amoxicillin/clavulanate, ciprofloxacin, azithromycin, and prednisone, he was hospitalized and treated with intravenous ceftazidime and tobramycin. His symptoms improved over the first few days of the hospitalization, but then he developed increased wheezing and dyspnea. His pulmonary function testing revealed a severe obstructive pattern, which improved with bronchodilator administration (from a forced expiratory volume in 1 second of $27 \%$ to $34 \%$ of predicted based on his height and age). He also had eosinophilia (13\%) documented while he was receiving the intravenous antibiotics. A chest radiograph revealed a slight right middle lobe infiltrate. He was treated with a two week course of oral prednisone, with rapid improvement in his symptoms. His pulmonary function improved within a week and his eosinophils normalized about a week off intravenous tobramycin. His chest radiograph then revealed clear lung fields.

In further work-up of the patient's recurrent eosinophilia, the possibility of a fungal sinus infection was proposed by an Infectious Disease consultant. Therefore, the patient underwent computed tomography studies of his sinuses that revealed bilateral opacification of the maxillary sinuses. A sinus biopsy was consistent with chronic sinusitis. The sinus specimen cultures revealed Alcaligenes xylosoxidans, but were negative for fungus and acid fast organisms. Because of the apparent worsening of his chronic clinical condition half a year after discontinuation of every other month TSI therapy, and given the presence of the Gram-negative organisms Serratia and Alcaligenes in his airways, the patient was restarted on every other month TSI.
He developed recurrent wheezing once his prednisone was discontinued. He was subsequently started on every other day prednisone, at a dose of $0.8 \mathrm{mg} / \mathrm{kg} /$ day, which helped control his wheezing. At 6 years TSI was discontinued as he was found to harbor Alcaligenes xylosoxidans in his deep throat culture, which was resistant to tobramycin.

He did well until $61 / 2$ years when he developed an upper respiratory infection with associated sinusitis and coughing. A throat culture demonstrated Pseudomonas aeruginosa for the first time since his early childhood for which he was prescribed oral ciprofloxacin and TSI. Within several days, he developed severe wheezing with an associated severe obstructive pattern on pulmonary function testing with eosinophilia (14\%). At that time, review of his medical record revealed a strong relationship between use of tobramycin and the patient's eosinophilia and bronchospasm (Tables 1 and 2). This prompted permanent discontinuation of the patient's TSI.

Over the subsequent 12 months, the patient did not demonstrate eosinophilia. He tolerated weaning off chronic prednisone by the age of 7 years. He continued to have elevated serum IgE levels, however was noted to be trending down while off TSI (Table 2). He has had recurrent mild bronchospasm, although he has not had further episodes of severe airway obstruction.

\section{Discussion}

Tobramycin associated recurrent eosinophilia with severe persistent bronchospasm has not been reported previously. The temporal sequence of the patient's signs and symptoms, after every administration of TSI or tobramycin intravenously, strongly suggest an adverse drug reaction (ADR).

An ADR is a noxious and unintended response to a drug at therapeutic doses [11] - in our patient, documented as eosinophilia and severe obstructive airway disease after every administration of tobramycin. The Naranjo scale confirms tobramycin as the highly probable cause of his ADR. This is a reliable (inter-raters $[r=0.92]$ and intra-

Table 2: Summary of TSI administration, pulmonary function, and hematologic profiles at 5 years and after

\begin{tabular}{|c|c|c|c|c|c|c|c|}
\hline & Off TSI & $\begin{array}{c}\text { Intravenous } \\
\text { tobramycin } \sim \text { I week }\end{array}$ & $\begin{array}{c}\sim \text { I weeks post } \\
\text { intravenous tobramycin }\end{array}$ & Off TSI & 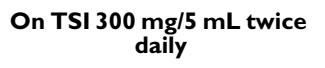 & Off TSI & Off TSI \\
\hline Age & 5 years & $5 \mathrm{I} / 2$ years & $5 \mathrm{I} / 2$ years & 6 years & $61 / 2$ years & 7 years & $71 / 2$ years \\
\hline Pulmonary Function & $\begin{array}{l}\text { FVC } 105 \% \\
\text { FEV, } 90 \%\end{array}$ & $\begin{array}{l}\text { FVC } 45 \% \\
\text { FEV }, 27 \%\end{array}$ & $\begin{array}{l}\mathrm{FVC} 76 \% \\
\mathrm{FEV}, 64 \%\end{array}$ & $\begin{array}{l}\text { FVC } 103 \% \\
\text { FEV, } 93 \%\end{array}$ & $\begin{array}{l}\mathrm{FVC} 49 \% \\
\mathrm{FEV}, 32 \%\end{array}$ & $\begin{array}{l}\text { FVC } 90 \% \\
\text { FEV }, 81 \%\end{array}$ & $\begin{array}{l}\mathrm{FVC} 91 \% \\
\mathrm{FEV}, 78 \%\end{array}$ \\
\hline WBC $\left(/ \mathrm{mm}^{3}\right)$ & 7.9 & 16 & 15.8 & NA & 17.7 & 13.5 & 9.9 \\
\hline Eosinophils (\%) & 2 & 13 & 1 & NA & 14 & 0 & 1 \\
\hline $\operatorname{IgE}(\mathrm{IU} / \mathrm{mL})$ & 123 & 165 & NA & NA & 205 & 306 & 166 \\
\hline
\end{tabular}

Abbreviations: TSI, tobramycin solution for inhalation; WBC, white blood cell count; FVC, forced vital capacity; FEV , forced expiratory volume in I second; NA, not available 
raters $[\mathrm{r}=0.91]$ reliability, $\mathrm{p}<0.001$ ) and valid (with consensual, content and concurrent validity) probability scale consisting of a simple questionnaire used to assess ADR [12]. Although his serum IgE remained elevated, there was no recurrence of eosinophilia after discontinuation of the TSI. He has had episodes of bronchospasm; however, not as severe as when he was receiving TSI. Furthermore, other emergent causes of his signs and symptoms (such as allergic bronchopulmonary aspergillosis and parasitemia) have been excluded.

Hypersensitivity reaction to tobramycin is a plausible explanation in our patient. This has been reported in an adult patient with CF who developed urticaria, pruritis, and difficulty of breathing after the third dose of intravenous tobramycin [13]. In our patient, hypersensitivity reaction is supported by his history of environmental allergies.

The patient might have benefited from a diagnostic bronchoscopy when he developed unexplained respiratory symptoms with associated eosinophilia. This procedure might have documented eosinophilia, Pseudomonas, fungus, or another infectious organism in the bronchoalveolar lavage fluid, and such findings could have guided his therapy.

There are in vitro data to suggest that aminoglycoside accumulation in non-CF cells is under regulation by effectors and inhibitors of the CF transmembrane conductance (CFTR) channel. This regulation is lost among CF cells with $\Delta \mathrm{F} 508$ mutations with inhibition of exocytosis since the CFTR channel is inoperable. This results in accumulation of aminoglycoside within the affected cells [14]. As patients exposed to higher drug concentrations are more at risk for ADR, it is possible that our patient had significant tissue accumulation of tobramycin leading to his symptoms.

Any drug has the potential to produce an ADR. Medical personnel taking care of children with complicated medical conditions such as CF, need to maintain a high index of suspicion in differentiating complications of the primary illness from an unexpected consequence of prescribed medications [15].

\section{Conclusion}

Hypersensitivity reaction should be considered in patients who develop recurrent eosinophilia and deterioration of pulmonary function following the use of tobramycin by inhalation or by intravenous administration.

\section{Competing interests}

The author(s) declare that they have no competing interests.

\section{Authors' contributions}

RS, EA, and RA wrote the case report. RA treated the reported patient, and edited the report. All authors read and approved the final manuscript.

\section{Acknowledgements}

This study was not funded.

Written consent was obtained from the patient's parents for publication of this report.

\section{References}

I. Prober CG, Walson PD, Jones J, Committee on Infectious Diseases and Committee on Drugs: Technical report: precautions regarding the use of aerosolized antibiotics. Pediatrics 2000, I06(6): E89.

2. Cooney GF, Lum BL, Tomaselli M, Fiel SB: Absolute bioavailability and absorption characteristics of aerosolized tobramycin in adults with cystic fibrosis. J Clin Pharmacol I994, 34(3):255-259.

3. Hoffmann IM, Rubin BK, Iskandar SS, Schechter MS, Nagaraj SK, Bitzan MM: Acute renal failure in cystic fibrosis: association with inhaled tobramycin therapy. Pediatr Pulmonol 2002, 34(5):375-377.

4. Edson RS, Brey RH, McDonald TJ, Terrell CL, McCarthy JT, Thibert J: Vestibular toxicity due to inhaled tobramycin in a patient with renal insufficiency. Mayo Clin Proc 2004, 79(9): I I 85- I I 9 I.

5. Spigarelli MG, Hurwitz ME, Nasr SZ: Hypersenstivity to inhaled TOBI ${ }^{\circledR}$ following reaction to gentamicin. Pediatr Pulmonol 2002, 33(4):3||-3|4.

6. Ramsey BW, Pepe MS, Quan JM, Otto KL, Montgomery AB, WilliamsWarren J, Vasiljev-K M, Borowitz D, Bowman CM, Marshall BC, Marshall $S$, Smith $A L$ : Intermittent administration of inhaled tobramycin in patients with cystic fibrosis. Cystic Fibrosis Inhaled Tobramycin Study Group. $N$ Engl J Med 1999, 340(I):23-30.

7. Gibson RL, Emerson J, McNamara S, Burns JL, Rosenfeld M, Yunker A, Hamblett N, Accurso F, Dovey M, Hiatt P, Konstan MW, Moss R, Retsch-Bogart G, Wagener J, Waltz D, Wilmott R, Zeitlin PL, Ramsey B, Cystic Fibrosis Therapeutics Development Network Study Group: Significant microbiological effect of inhaled tobramycin in young children with cystic fibrosis. Am J Respir Crit Care Med 2003, I 67(6):84I-849.

8. Meyers BR: Clinical study of the use of the new aminoglycoside tobramycin for therapy of infections due to gram-negative bacteria. Med J Aust 1977, 2(3 Pt 2 Suppl):38-40.

9. Jones LM, Miller S: Bacterial peritonitis. Protecting the highrisk patient. Am Surg 1984, 50(7):358-36I.

10. Hannah BA, Kimmel PL, Dosa S, Turner ML: Vancomycin-induced toxic epidermal necrolysis. South Med J 1990, 83(6):720-722.

II. Edwards IR, Aronson JK: Adverse drug reactions: definitions, diagnosis, and management. Lancet 2000, 356(9237): I 255-1259.

12. Naranjo CA, Busto U, Sellers EM, Sandor P, Ruiz I, Roberts EA Janecek E, Domecq C, Greenblatt DJ: A method for estimating the probability of adverse drug reactions. Clin Pharmacol Ther 1981, 30(2):239-245.

13. Schretlen-Doherty JS, Troutman WG: Tobramycin-induced hypersensitivity reaction. Ann Pharmacother 1995, 29(78):704-706

14. Quesnel LB, Jaran AS, Braganza JM: Antibiotic accumulation and membrane trafficking in cystic fibrosis cells. J Antimicrob Chemother 1998, 4 I(2):215-22I.

15. Parmar JS, Nasser S: Antibiotic allergy in cystic fibrosis. Thorax 2005, 60(6):517-520.

\section{Pre-publication history}

The pre-publication history for this paper can be accessed here:

http://www.biomedcentral.com/1471-2431/7/11/prepub 